\title{
Determination of Eicosapentaenoic, Docosahexaenoic, and Arachidonic Acids in Human Plasma by High-Performance Liquid Chromatography with Electrochemical Detection
}

\author{
Akira Kotani, ${ }^{\dagger}$ Mizuki Watanabe, Kazuhiro Yamamoto, Fumiyo Kusu, and Hideki Hakamata \\ Department of Analytical Chemistry, School of Pharmacy, Tokyo University of Pharmacy and Life Sciences, \\ 1432-1 Horinouchi, Hachioji, Tokyo 192-0392, Japan
}

\begin{abstract}
A high-performance liquid chromatography with electrochemical detection (HPLC-ECD) system was developed for the simultaneous determination of eicosapentaenoic acid (EPA), docosahexaenoic acid (DHA), and arachidonic acid (AA) in human plasma. In the present HPLC-ECD system, EPA, DHA, and AA were separated using a reverse-phase $\mathrm{C}_{30}$ column and detected based on the voltammetric reduction of 3,5-di-tert-butyl-1,2-benzoquinone (DBBQ). Chromatographic peak areas were proportional to the concentration of EPA, DHA, and AA from $0.75 \mu \mathrm{M}$ to $0.1 \mathrm{mM}(r>0.998)$. The concentrations of EPA, DHA, and AA in plasma from healthy human subjects after overnight fasting were determined, and the ratio of EPA to AA was obtained by the present HPLC-ECD method, which required $40 \mu \mathrm{L}$ of human plasma and a simple procedure of sample preparation using diethyl ether extraction. Moreover, changes in EPA, DHA, and AA concentrations in a human subject were monitored before and after fish oil supplement administration by the present HPLC-ECD system.
\end{abstract}

Keywords Electrochemical detection, HPLC, plasma, eicosapentaenoic acid, docosahexaenoic acid, arachidonic acid

(Received April 19, 2016; Accepted May 27, 2016; Published September 10, 2016)

\section{Introduction}

Eicosapentaenoic acid (EPA) and docosahexaenoic acid (DHA) classified into $\omega$-3 polyunsaturated fatty acids, are important for growth and development. ${ }^{1,2}$ EPA and DHA intake reduces overall mortality and sudden death in patients with coronary heart diseases. ${ }^{3}$ EPA and DHA inhibit pro-inflammatory eicosanoid formation, reduce the susceptibility of ventricular arrhythmia, retard the growth of atherosclerotic plaque, and promote nitric oxide-induced endothelial relaxation, ${ }^{4,5}$ thereby playing important roles in the prevention and treatment of inflammatory diseases, cardiovascular diseases, and cancer. . $^{1,2,6-10}$ In addition, there are various reports that show that EPA and DHA have the potential to exert beneficial effects in patients with kidney disease. ${ }^{11}$ Furthermore, dietary intake of DHA and EPA has been recommended to reduce the risk of coronary heart disease by the Food and Drug Administration (FDA). ${ }^{12}$

Recently, it has been reported that the ratio of EPA to arachidonic acid (AA) in plasma can be a biomarker for cardiovascular events in general clinical practice. ${ }^{13-15}$ In addition, patients with chronic kidney diseases (CKD) are at an increased risk of cardiovascular disease (CVD); this may be explained by both classic risk factors and novel risk factors specific to CKD, such as inflammation with wasting, CKDmineral and bone disorders, and renal anemia. ${ }^{16}$ Thus, many large-scale clinical trials have been performed to examine the

$\dagger$ To whom correspondence should be addressed.

E-mail: kotani@toyaku.ac.jp detailed pharmacological effects and clinical efficacy of EPA and/or DHA in the fields of cardiology ${ }^{17,18}$ and nephrology..$^{19,20}$ To help the effective progression of these clinical studies, the use of a simple, selective, and sensitive analytical method for determining EPA, DHA, and AA should be of significant importance.

Gas chromatography with flame ionization detection (GC-FID), ${ }^{21}$ GC with mass spectrometry (GC-MS), ${ }^{22}$ high-performance liquid chromatography with UV detection (HPLC-UV), ${ }^{23}$ HPLC with fluorescent detection (HPLC-FL), ${ }^{24}$ LC with mass spectrometry (LC-MS), ${ }^{25}$ and LC-tandem mass spectrometry (LC-MS/MS) ${ }^{26,27}$ have been applied to determine EPA, DHA, and AA. However, in GC-MS, HPLC-UV, HPLC$\mathrm{FL}$, and LC-MS, the cleanup and derivatization procedures are essential for the sensitive and selective determination of EPA, DHA and AA in real sample analysis. Thus, using simpler sample preparation procedures, a sensitive and selective analytical method for determining EPA, DHA, and AA without derivatization is highly desirable from the point of view of clinical laboratory studies. We have developed a voltammetric method using 3,5-di-tert-butyl-1,2-benzoquinone (DBBQ) for determining plasma free fatty acids (FFAs) in HPLC with electrochemical detection (HPLC-ECD), and it was shown to be quite sensitive and selective for acid determination without derivatization. ${ }^{28}$ However, the simultaneous determination of EPA, DHA, and FFAs is difficult in the reported isocratic elution HPLC-ECD because the hydrophobic properties among EPA, DHA and main plasma FFAs such as saturated and monounsaturated FFAs are so different. If HPLC-ECD based on voltammetric reduction of DBBQ is applied to determine 
EPA, DHA, and AA by the development of a new system, HPLC-ECD can be fabricated as a less expensive analytical system compared with LC-MS and LC-MS/MS.

In the present study, we fabricate an HPLC-ECD system for determining EPA, DHA, and AA in human plasma. Moreover, the present HPLC-ECD is applied to determine the ratio of EPA to AA (EPA/AA ratio) in human subjects and to examine timeconcentration profiles of EPA, DHA, and AA in a human subject after fish oil supplement administration.

\section{Experimental}

\section{Protocol for human subjects}

Three healthy subjects (A: male, age 40 years; B: male, 46 years; C: female, age 23 years) were used in our study upon the receipt of their informed consent. The protocol and the subject's informed consent were approved by the Institutional Human Research Committee of Tokyo University of Pharmacy and Life Sciences before the commencement of the trial (approval number: 14-06).

After overnight fasting, a blood specimen of $0.1 \mathrm{~mL}$ was obtained by a finger pricked with a lancing device (MS-GN02, Terumo, Tokyo, Japan). Heparinized blood specimen was centrifuged at $3000 \mathrm{rpm}$ for $10 \mathrm{~min}$, and then plasma was collected in another test tube. Moreover, subject A had fasted for $11 \mathrm{~h}$ and ingested two capsules of fish oil, which consisted of $273 \mathrm{mg}$ fish oil containing total EPA of $40 \mathrm{mg}$ and DHA of $27 \mathrm{mg}$ in each capsule, (Nature Made Fish Oil Supplements, Otsuka Pharmaceutical, Tokyo, Japan). Blood specimen of subject A was taken before and 0.5, 1, 1.5, 2, 2.5, 3, 3.5, 4, 5, and $6 \mathrm{~h}$ after ingestion. Plasma was stored at $-30^{\circ} \mathrm{C}$ until analysis.

\section{Sample preparation}

Forty microliters of control human serum (Model I, Wako Pure Chemical Industries, Osaka, Japan) and human plasma were mixed with 0.4-mL diethyl ether, and EPA, DHA, and AA in plasma were extracted into the diethyl ether phase. The extraction by diethyl ether and collection of supernatant were repeated three times. The collected diethyl ether was dried completely by nitrogen stream to obtain the lipid residue, which was then dissolved in a $20-\mu \mathrm{L}$ undecanoic acid $(0.2 \mathrm{mM}$ in acetonitrile and water $=80: 20, \mathrm{v} / \mathrm{v}$ ) as internal standard (I.S.), followed by an addition of a $20-\mu \mathrm{L}$ mixture of acetonitrile and water $(80: 20, \mathrm{v} / \mathrm{v})$ to prepare a test solution. The test solution was filtered through a membrane filter (pore size, $0.45 \mu \mathrm{m}$ ), and then $5 \mu \mathrm{L}$ of the filtrate was injected into the HPLC-ECD system.

\section{HPLC-ECD system and chromatographic conditions}

Chromatography was performed using an AI-12 pump (Flom, Tokyo, Japan) equipped with a $7725 \mathrm{i}$ syringe loading sample injector valve ( $5 \mu \mathrm{L}$ loop, Rheodyne, Cotati, CA). EPA, DHA, and AA were separated on a reverse phase column, Develosil C30-XG-3 $(250 \times 1.0 \mathrm{~mm}$, i.d., particle size $3 \mu \mathrm{m}$, Nomura Chemical, Aichi, Japan) by isocratic elution with a mixture of acetonitrile and water $(80: 20, \mathrm{v} / \mathrm{v})$ as a mobile phase. The flow rate of the mobile phase was set at $30 \mu \mathrm{L} \mathrm{min}{ }^{-1}$, and the column temperature was set at $30^{\circ} \mathrm{C}$. Via the MT1XCPK micro volume connector (VICI, Houston, TX), the eluate was allowed to merge with the stream of DBBQ solution, which was a mixture of acetonitrile and water (80:20, v/v) containing $2 \mathrm{mM}$ DBBQ and $20 \mathrm{mM}$ lithium perchlorate, to be mixed together in a mixing tube, and then the EPA, DHA, and AA were detected at

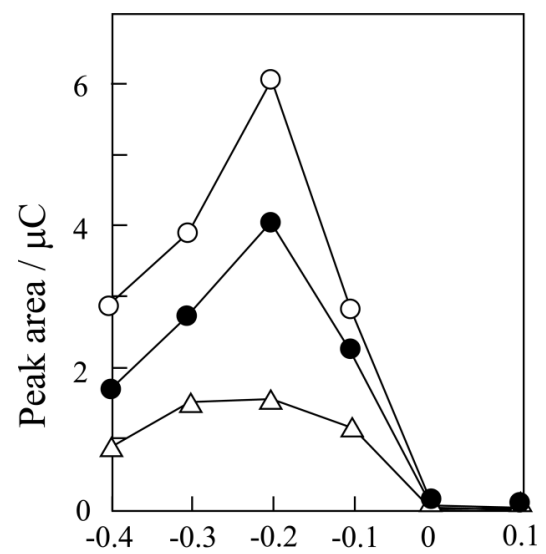

Potential / V vs. Ag/AgCl

Fig. 1 Potential dependencies of the chromatographic peak areas for EPA ( $\bigcirc)$, DHA $(\bullet)$, and AA $(\triangle)$, respectively. Five microliters of EPA, DHA, and AA standard at $50 \mu \mathrm{M}$ each were injected into the HPLC-ECD system.

$-0.2 \mathrm{~V}$ vs. $\mathrm{Ag} / \mathrm{AgCl}$ in an $\mathrm{LC}-4 \mathrm{C}$ electrochemical detector (BAS, Tokyo, Japan) equipped with a radial flow electrochemical cell (BAS) using a glassy carbon working electrode, an $\mathrm{Ag} / \mathrm{AgCl}$ reference electrode, and a stainless-steel counter electrode. The DBBQ solution was made to flow at $100 \mu \mathrm{L} \mathrm{min}{ }^{-1}$ using an AI-12 pump.

\section{Results and Discussion}

HPLC-ECD for determining EPA, DHA, and AA

Chromatograms of a standard solution containing EPA, DHA, and AA were measured at various applied potentials to select an optimal applied potential for the sensitive monitoring of EPA, DHA, and AA, and the chromatographic peak areas of EPA, DHA, and AA were plotted against the applied potential. As shown in Fig. 1, the chromatographic peak areas give a maximum at $-0.2 \mathrm{~V}$ vs. $\mathrm{Ag} / \mathrm{AgCl}$, and based on this result, the applied potential of the working electrode was fixed at $-0.2 \mathrm{~V}$ vs. $\mathrm{Ag} / \mathrm{AgCl}$ for the monitoring of EPA, DHA, and $\mathrm{AA}$. In the present HPLC-ECD system, the reduction current of DBBQ was observed as a chromatographic background current. Thus, because chromatographic peaks of acids appeared based on the difference between the background current and the reduction prepeak current of DBBQ caused by acid, ${ }^{29}$ the peak areas of EPA, DHA, and AA are reduced at more negative potential than $-0.2 \mathrm{~V}$ vs. $\mathrm{Ag} / \mathrm{AgCl}$ as shown in Fig. 1 .

A typical chromatogram of a standard solution containing EPA, DHA, and AA is shown in Fig. 2A. The chromatographic peaks of EPA, DHA, and AA were observed within $30 \mathrm{~min}$, where the resolutions $\left(R_{\mathrm{s}}\right)$ between the EPA and DHA, and DHA and AA were 5.3 and 2.7 , respectively. The chromatographic peak areas of EPA, DHA, and AA were found to be proportional to each analyte concentration, ranging from $0.75 \mu \mathrm{M}$ to $0.1 \mathrm{mM}(r>0.998)$. The relative standard deviation (RSD) of the chromatographic peak areas for the standard solutions of EPA, DHA, and AA at $50 \mu \mathrm{M}$ were 2.4, 3.3, and $2.9 \%$, respectively. The detection limits $(S / N=3$, signal-tonoise ratio of $3: 1$ ) of the EPA, DHA, and AA were $0.13,0.14$, and $0.23 \mu \mathrm{M}$, respectively. The quantitative sensitivity was compared with other available methods for determining EPA, 


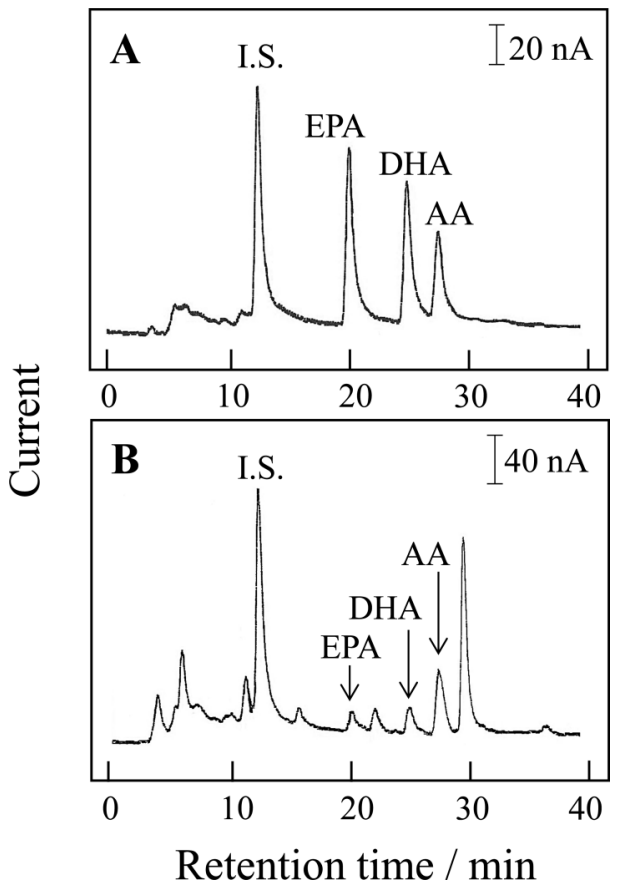

Fig. 2 Chromatogram of (A) standard solution (50 $\mu \mathrm{M}$ EPA, DHA, AA, and undecanoic acid (I.S.)) and (B) control human serum.

DHA, and AA. The quantitation limits of EPA by GC-MS based on methyl ester derivatization, ${ }^{22}$ HPLC-UV based on $p$-bromophenacyl bromide derivatization, ${ }^{23} \mathrm{LC}$-MS/MS without derivatization, ${ }^{26} \mathrm{LC}-\mathrm{MS}$ based on 7-(N,N-dimethylaminosulfonyl)4-(aminoethyl) piperazino-2,1,3-benzoxadiazole (DBD-PZ-NH ${ }_{2}$ ) derivatization, ${ }^{25}$ and HPLC-FL based on 2-(12-oxobenzo[b]acridin-5 (12H)-yl)-ethyl-4-toluenesulfonate (BAETS) derivatization $^{24}$ were $51.55 \mu \mathrm{M}, 1.63 \mu \mathrm{M}, 0.33 \mu \mathrm{M}, 0.1 \mu \mathrm{M}$, and $4.73 \mathrm{nM}$, respectively. The present HPLC-ECD for determining EPA, DHA, and AA was highly sensitive in comparison with GC-MS ${ }^{22}$ and HPLC-UV, ${ }^{23}$ and the present HPLC-ECD provided similar sensitivity to LC-MS/MS. $^{26}$ However, the present HPLC-ECD was less sensitive in comparison with the LC/MS based on DBD-PZ- $\mathrm{NH}_{2}$ derivatization $^{25}$ and the HPLC-FL based on BAETS derivatization. ${ }^{24}$ In an analysis of biological samples by LC/MS and HPLC-FL, the procedure of derivatization and cleanup of the samples is sometimes complicated and time-consuming. Thus, the present HPLC-ECD, which does not require a derivatization procedure to detect EPA, DHA, and AA, would be a favorable method in an analysis when using biological samples.

\section{Determination of the ratio of EPA to AA in human plasma}

A chromatogram for control human serum is shown in Fig. 2B. The chromatographic peaks of EPA, DHA, and AA are clearly separated with other unknown peaks derived from biological compounds in the control human serum, indicating that the present HPLC-ECD system is selective for determining EPA, DHA, and AA in blood samples. As shown in Table S1 (Supporting Information), the concentrations of EPA, DHA, and $\mathrm{AA}$ in control human serum are 10.4, 16.4, and $129 \mu \mathrm{M}$, respectively, and the repeatability is less than $3.3 \% \operatorname{RSD}(n=6)$. The recovery rate $(n=6)$ of the EPA, DHA, and AA for the spiked control human serum was 99.2, 101.1, and 101.6\%, respectively, and its RSD was less than $5.5 \%$. These results
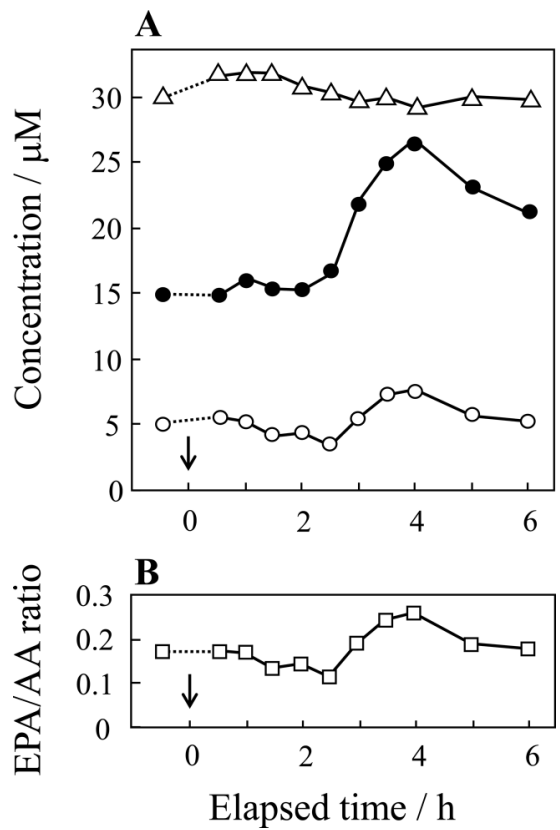

Fig. 3 Time-concentration profiles of $(A)$ EPA $(\bigcirc)$, DHA $(\bullet)$, and AA $(\triangle)$ and (B) EPA/AA ratio $(\square)$ before and after fish oil supplement administration. Arrows in the figures show the point of fish oil supplement administration, i.e., the elapsed time is $0 \mathrm{~h}$ on these profiles.

show that the HPLC-ECD provided accurate and precise measurements of EPA, DHA, and AA in human serum and plasma.

Table S2 (Supporting Information) shows concentrations of EPA, DHA, and AA and the EPA/AA ratio in plasma from healthy human subjects subsequent to fasting for $11 \mathrm{~h}$. The EPA/AA ratio of healthy human subjects $\mathrm{A}, \mathrm{B}$, and $\mathrm{C}$ was 0.14 , 0.13 , and 0.11 , respectively. The EPA/AA ratio of healthy human subjects studied was within the range of reported EPA/ AA ratios of healthy Japanese who are living in urban areas when the EPA/AA ratio of each subject was compared with that of the same generation. ${ }^{17,30}$ Thus, our results of EPA/AA ratio in human plasma obtained by the present HPLC-ECD system are appropriate.

Application to obtain time-concentration profiles of EPA, DHA, and $A A$ after administration of fish oil supplement

To show that the present HPLC-ECD system was applied to the human pharmacokinetic analysis of EPA, DHA, AA, and EPA/AA ratio, the time-concentration profiles of EPA, DHA, and AA and EPA/AA ratio before and after fish oil supplement administration in subject $\mathrm{A}$ are examined. As shown in Fig. 3, the concentrations of EPA and DHA in plasma were increased at $2-3 \mathrm{~h}$ after the fish oil supplement ingestion, reaching a maximum at $4 \mathrm{~h}$. Thereafter, EPA and DHA concentrations were reduced. EPA/AA ratio also reached a maximum at $4 \mathrm{~h}$ after fish oil supplement administration.

To date, there have been other reports regarding the concentration of EPA and DHA in plasma after administration of fish oil or EPA-related medicine. The dosages of EPA and DHA in these previous studies ${ }^{31-34}$ were about $10-50$ times higher than our dosage. In addition, a single time measurement for determining high concentration levels of EPA, DHA, and AA was performed for clinical purposes. In the present study, time-concentration profiles of EPA, DHA, and AA were obtained regardless of the low dosage of EPA and DHA in fish 
oil, which are the same level as the daily administration. We have obtained these results because the present HPLC-ECD system is sufficiently sensitive for determining EPA, DHA, and AA in plasma. Moreover, the present method has an advantage from the viewpoint of bioethics to reduce the burden in human patients because plasma EPA, DHA, and AA can be determined using $40 \mu \mathrm{L}$ plasma which can be obtained by a lancing device. Thus, it was found that the present method, which provided accurate and precise quantitative results in plasma EPA, DHA, and AA determination, can be utilized and contribute to the progress of clinical studies concerned with plasma EPA, DHA, and AA.

\section{Conclusions}

The present HPLC-ECD system could be a powerful analytical method not only for determining the EPA/AA ratio in plasma but also for examining the time-concentration profiles of EPA, DHA, and AA. This is because it is able to detect EPA, DHA, and AA without the derivatization of acids and the procedure for the sample preparation using a small blood sample would be facile. Thus, the present HPLC-ECD method would help in pathophysiological research to show the involvement of EPA, DHA, and AA in cardiovascular diseases and chronic kidney diseases

\section{Acknowledgements}

This research was supported in part by a Grant-in-Aid for Scientific Research C (No. 26410161) from the Ministry of Education, Culture, Sports, Science and Technology of Japan.

\section{Supporting Information}

The chemicals, the recovery test, and the determination results of human control serum and plasma samples are shown in Supporting Information. This material is available free of charge on the Web at http:/www/jsac.or.jp/analsci/.

\section{References}

1. C. A. Drevon, Nutr. Rev., 1992, 50, 38.

2. A. P. Simopoulos, Am. J. Clin. Nutr., 1991, 54, 438.

3. H. C. Bucher, P. Hengstler, C. Schindler, and G. Meier, Am. J. Med., 2002, 112, 298.

4. P. M. Kris-Etherton, W. S. Harris, and L. J. Appel, Arterioscler. Thromb. Vasc. Biol., 2003, 23, E20.

5. P. C. Calder, Clin. Sci., 2004, 107, 1.

6. P. C Calder, Am. J. Clin. Nutr., 2006, 83(Suppl.), 1505S.

7. P. M. Kris-Etherton, W. S. Harris, and L. J. Appel, Circulation, 2002, 106, 2747.

8. J. L. Breslow, Am. J. Clin. Nutr., 2006, 83(Suppl.), 1477S.

9. M. F. Leitzmann, M. J. Stampfer, D. S. Michaud, K. Augustsson, G. C. Colditz, W. C. Willett, and E. L. Giovannucci, Am. J. Clin. Nutr., 2006, 80, 204.

10. C. E. Roynette, P. C. Calder, Y. M. Dupertuis, and C. Pichard, Clin. Nutr., 2004, 23, 139.

11. R. G. Fassett, G. C. Gobe, J. M. Peake, and J. S. Coombes,
Am. J. Kidney Dis., 2010, 56, 728.

12. U. S. Food and Drug Administration, "FDA Announces Qualified Health Claims for Omega-3 Fatty Acids", FDA News Release, 2004, P04, 89.

13. P. B. Adams, S. Lawson, A. Sanigorski, and A. J. Sinclair, Lipids, 1996, 31(Suppl.), S157.

14. H. Ohnishi and Y. Saito, J. Atheroscler. Thromb., 2013, 20 , 861.

15. M. Yokoyama, H. Origasa, M. Matsuzaki, Y. Matsuzawa, Y. Saito, Y. Ishikawa, S. Oikawa, J. Sasaki, H. Hishida, H. Itakura, T. Kita, A. Kitabatake, N. Nakaya, T. Sakata, K. Shimada, and K. Shirato, Lancet, 2007, 369, 1090.

16. T. Shoji, R. Kakiya, T. Hayashi, Y. Tsujimoto, M. Sonoda, H. Shima, K. Mori, S. Fukumoto, H. Tahara, A. Shioi, T. Tabata, M. Emoto, Y. Nishizawa, and M. Inaba, Am. J. Kidney Dis., 2013, 62, 568.

17. N. Yanagisawa, K. Shimada, T. Miyazaki, A. Kume, Y. Kitamura, R. Ichikawa, H. Ohmura, T. Kiyanagi, M. Hiki, K. Fukao, K. Sumiyoshi, K. Hirose, R. Matsumori, H. Takizawa, K. Fujii, H. Mokuno, N. Inoue, and H. Daida, $J$. Atheroscler. Thromb., 2010, 17, 285.

18. T. Domei, H. Yokoi, S. Kuramitsu, Y. Soga, T. Arita, K. Ando, S. Shirai, K. Kondo, K. Sakai, M. Goya, M. Iwabuchi, M. Ueeda, and M. Nobuyoshi, Circ. J., 2012, 76, 423.

19. J. V. Jr. Donadio, T. S. Larson, E. J. Bergstralh, and J. P. Grande, J. Am. Soc. Nephrol., 2001, 12, 791.

20. R. J. Hogg, L. Fitzgibbons, C. Atkins, N. Nardelli, and R. C. Bay, Clin. J. Am. Soc. Nephrol., 2006, 1, 1167.

21. H. M. Liebich, C. Wirth, and B. Jakober, J. Chromatogr. B, 1991, 572, 1

22. D. M. Pereira, J. Vinholes, P. G. de Pinho, P. Valentao, T. Mouga, N. Teixeira, and P. B. Andrade, Talanta, 2012, 100, 391.

23. C. Rustichelli, R. Avallone, E. Campioli, D. Braghiroli, C. Parenti, and M. Baraldi, J. Food Lipids, 2009, 16, 422.

24. N. Jing, J. Shi, G. Li, Z. Sun, and J. You, Food Res. Int., 2012, 48, 155.

25. Y. Tsukamoto, T. Santa, H. Yoshida, H. Miyano, T. Fukushima, K. Hirayama, K. Imai, and T. Funatsu, Biomed Chromatogr., 2006, 20, 358.

26. M. Aslan, G. Celmeli, F. Ozcan, and A. Kupesiz, Clin. Exp. Med., 2015, 15, 397.

27. C. Pettinella, S. H. Lee, F. Cipollone, and I. A. Blair, $J$. Chromatogr. B, 2007, 850, 168.

28. A. Kotani, T. Kotani, N. Ishii, H. Hakamata, and F. Kusu, J. Pharm. Biomed. Anal., 2014, 97, 47.

29. A. Kotani, Y. Miyaguchi, D. Harada, and F. Kusu, Anal. Sci., 2003, 19, 1473.

30. T. Hasegawa and M. Oshima, J. Jpn. Atheroscler. Soc., 1998, 25, 283.

31. W. S. Harris, S. A. Varvel, J. V. Pottala, G. R. Warnick, and J. P. McConnell, J. Clin. Lipidol., 2013, 7, 433.

32. M. Rondanelli, A. Giacosa, A. Opizzi, C. Pelucchi, C. La Vecchia, G. Montorfano, M. Negroni, B. Berra, P. Politi, and A. M. Rizzo, J. Am. Coll. Nutr., 2010, 29, 55.

33. G. Querques, P. Benlian, B. Chanu, C. Portal, G. Coscas, G. Soubrane, and E. H. Souied, Eur. J. Ophthalmol., 2009, 19, 100.

34. C. Galli, F. M. Maggi, P. Risé, and C. R. Sirtori, Br. J. Clin. Pharmacol., 2012, 74, 60. 